\title{
Mudança climática e a pegada ecológica dos resíduos sólidos da cidade de Parintins
}

O presente trabalho tem como objetivo calcular a pegada ecológica (Ecological Footprint Method- EFM) dos resíduos sólidos urbanos (RSU) da cidade de Parintins de 2011 e 2013. Os dados em toneladas foram encontrados na Secretaria Municipal de Limpeza Pública (SEMOSP) e Secretaria Municipal de Meio Ambiente (SEMMA). Posteriormente, se aplicou a metodologia da pegada ecológica que quantifica os resíduos gerados em $\mathrm{CO} 2$ e CH4, principais gases do aquecimento global. Depois, aplica-se o fator de equivalência de 1,37 referentes a bioprodutividade global de terras de energia para padronizar em global hectare (gha), a medida da pegada ecológica. 0 per capita/ano (0,3057 e 0,3763 gha) para os habitantes de Parintins demonstram estar aumentando a pegada ecológica dos RSU. A pegada ecológica agrega vários dados para completar sua matriz de análise. Se associarmos um problema ambiental, como a produção de resíduos sólidos urbanos, com a pegada ecológica, esta conseguirá quantificar ou mensurar sobre a pressão causada ao meio ecológico por nossas ações. Assim podemos acompanhar a mudança ao longo do tempo e comparar com outros espaços urbanos por meio deste indicadorRessalta-se que ainda são raras as pesquisas em cidades amazônicas por meio desse indicador de sustentabilidade. Porém, os resíduos sólidos urbanos por se constituírem num grande problema ambiental, cálculos como estes devem auxiliar na mensuração do impacto ecológico das sociedades urbanas. Por conseguinte, ao quantificarmos por meio de um indicador de sustentabilidade a produção RSU, observou-se a necessidade urgente de acompanhamento dos impactos gerados em outras cidades amazônicas.

Palavras-chave: Amazônia; Consumo; Sustentabilidade.

\section{Global warming and ecological footprint solid wast of Parintins (AM)}

\begin{abstract}
The present work has with the objective calculate the ecological footprint (Ecological Footprint Method- EFM) of municipal solid waste (MSW) the city of Parintins, 2011 and 2013. The data in tonnes were found in the Municipal Public Cleaning (SEMOSP) and Municipal Environment (SEMMA). Subsequently, if applied the methodology of ecological footprint which quantifies the residues generated CO2 and $\mathrm{CH} 4$, the main global warming gas. After, applies the 1.37 equivalence factor related to bioproductivity global energy land to standardize on global hectare (gha), the measure of the ecological footprint. The per capita / year ( 0.3057 and 0.3763 gha) to the in habitants of Parintins be shown increasing the ecological footprint of MSW. The ecological footprint aggregates multiple data to complete its analysis matrix. If we associate an environmental problem, as the production of municipal solid waste, the ecological footprint, you will be able to quantify or measure of the pressure caused to the ecological environment for our actions. So we can follow the change over time and compare to other urban spaces through this indicator. It is noteworthy that are still rare research in Amazonian cities through this indicator sustainability. However, municipal waste by itself constitute a major environmental problem, calculations like these should assist in measuring the ecological impact of urban societies.Therefore, when we quantify through a window sustainability MSW production, it was noted the urgent need to monitoring imapctos generated in other Amazonian cities.
\end{abstract}

Keywords: Amazonia; Consumption; Sustainability.

Topic: Meteorologia, Climatologia e Mudanças Climáticas

Reviewed anonymously in the process of blind peer.

\section{Alem Silvia Marinho dos Santos}

Universidade do Estado do Amazonas, Brasil

http://lattes.cnpq.br/4380195068604848

alemsilvia@gmail.com

\section{Edinelza Macedo Ribeiro}

Universidade do Estado do Amazonas, Brasil http://lattes.cnpq.br/5385395813417606

ediribeiro27@hotmail.com
Received: xx/03/2016

Approved: xx/08/2016

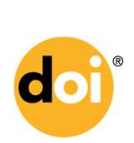

DOI: 10.6008/SPC2179-6858.2016.003.0007
Referencing this:

SANTOS, A. S. M.; RIBEIRO, E. M.. Mudança climática e a pegada ecológica dos resíduos sólidos da cidade de Parintins. Revista IberoAmericana de Ciências Ambientais, v.7, n.3, p.82-89, 2016. DOI: http://doi.org/10.6008/SPC2179-6858.2016.003.0007 


\section{INTRODUÇÃO}

A busca pela sociedade sustentável tornou-se o novo paradigma da ciência atual. Muitos trabalhos acadêmicos voltam-se para a elaboração de teorias, modelos, esquemas e quantificação daquilo que deve ser considerado ecologicamente correto. Assim se reconhece as novas exigências nas questões relacionadas ao meio ambiente. A Terra entrou num período de mudanças ambientais globais, devido aumento significativo da população humana mundial e do consumo produzido por esta, "o espaço físico terrestre disponível pode não ser suficiente para nos sustentar (e aos outros seres vivos)" (WWF, 2010). É nesse sentido que conceitos como sustentabilidade passaram a ser usados frequentemente

A palavra sustentabilidade vem do latim "sustentare" que significa suster, sustentar, suportar, conservar em bom estado, manter, resistir. Sustentável é tudo aquilo que é capaz de ser suportado (SICHÉ et al., 2007). É o limite de resiliência de nosso Planeta, dos ecossistemas terrestres.

Índices ou indicadores funcionam como um panorama do momento atual. Devido à complexidade ambiental podem deixar escapar certos fenômenos do sistema. Mas podem contribuir na avaliação deste sistema, sobretudo urbano, e orientar na organização de estratégias futuras por meio de cenários.

Entretanto, estudos prospectivos ou cenarização não são uma constante no Brasil. País em que a cultura do planejamento quase que se resume a elaboração e engavetamento de planos. $O$ estudo do futuro não poderia ser algo pertencente a sua cultura (RIBEIRO, 2013). Como mal se planejam os primeiros passos, um mês ou um ano, não é possível pensar que se planejem os anos que virão ou mesmo décadas. Na questão dos resíduos sólidos urbanos, é uma questão urgente seus cenários por meio de um indicador de sustentabilidade como a pegada ecológica.

Em uma análise superficial, índice e indicador possuem o mesmo significado, porem o primeiro é o mais complexo porque "é o valor agregado final de todo um procedimento de cálculo onde se utilizam, inclusive indicadores como variáveis que o compõem" (SICHE, et al., 2007). Entretanto, o indicador também utiliza dados agregados, por conseguinte, alguns autores (ANDRADE, 2006; BELLEN, 2006; PARENTE, 2007) consideram a pegada ecológica como tal e assim concordamos.

A pegada ecológica agrega vários dados para completar sua matriz de análise. Se associarmos um problema ambiental, como a produção de resíduos sólidos urbanos, com a pegada ecológica, esta conseguirá quantificar ou mensurar sobre a pressão causada ao meio ecológico por nossas ações. Assim podemos acompanhar a mudança ao longo do tempo e comparar com outros espaços urbanos por meio deste indicador

A Política Nacional de Resíduos Sólidos (2010), regulamentada pelo decreto no 7.404/10, institui no país uma nova maneira de tratarmos esse grave problema ambiental. A referida lei apresenta diversos pontos importantes para a gestão e o gerenciamento de resíduos sólidos respeitando-se, prioritariamente, a seguinte ordem: não geração, redução, reutilização, reciclagem, tratamento dos resíduos sólidos e pôr fim à disposição final ambientalmente adequada em aterros sanitários. 
O método de análise deste trabalho de cálculo RSU produzido em Parintins se constitui nas etapas para alcançar o resultado da pegada ecológica, dado em área bioprodutiva necessária para absorver determinados usos do meio ambiente, sempre expressa em global hectare (GHA). Por conseguinte, tanto a Pegada Ecológica (que representa a procura por recursos) como a Biocapacidade (que representa os recursos disponíveis) são expressas em unidades de hectares globais (GHA). Um hectare global é um hectare com capacidade mundial média para produzir recursos e absorver resíduos (PLANETA VIVO, 2010).

Neste sentido, como esse indicador tem a capacidade de transformar, por meio da quantificação do RSU (toneladas) o seu total em CO2 e CH4 (GEE)l, pode-se também inferir sobre o aquecimento global e as cidades amazônicas. Portanto, o objetivo deste estudo é calcular a pegada ecológica dos resíduos sólidos da cidade de Parintins nos anos de 2011 e 2013, referentes as emissões de $\mathrm{CO}_{2}+\mathrm{CH}_{4}$, produzidos pelos moradores da área urbana.

\section{METODOLOGIA}

A pegada ecológica ( $P E$ ) estabelece relações de dependência entre as atividades humanas e os recursos naturais necessários para a realização das mesmas e para a absorção dos resíduos gerados (DIAS, 2002). Constitui-se numa metodologia de contabilização ambiental criada por Wackernagel et al. (1996) e baseia-se no conceito de capacidade de carga.

A pegada ecológica é gerada a partir da pressão do homem sobre o meio ambiente, ou seja, tudo que gera impacto, danos deixados pela atividade humana e que podemos medir e calcular. Entretanto, a metodologia operacionaliza a capacidade de carga de maneira inversa. Dessa maneira, "a ferramenta deve perguntar: qual a área de terra ou mar bioprodutiva necessária para suprir determinada população sem prejuízo ao ecossistema natural?" (FIRMININO et al., 2009).

Ao final das etapas de contabilização ambiental, a metodologia expressa seu resultado em hectares que por meio do fator de equivalência. Este varia de acordo com cada uso do espaço de áreas bioprotutivas da Terra. Os espaços ecológicos utilizados na PE podem ser divididos em duas categorias: terrenos e consumos.

Na categoria de terrenos, os espaços ecológicos estão subdivididos em terra produtiva (pasto, cultivo, floresta), mar produtivo, áreas de energia fóssil e área construída (urbanizada) e biodiversidade. Na categoria de áreas de consumo devem-se considerar os itens que mais pressionam a natureza: alimentação (vegetais e carnes); habitação (casas e apartamentos); transporte (público e privado); bens de consumo (papel, maquina, roupa, etc.) e serviços (bancos, restaurantes, aeroportos entre outros).

Como neste trabalho iremos calcular a pegada ecológica do resíduo sólido urbano, o objetivo é medir a biocapacidade em hectare global do planeta que pode ser utilizado pela humanidade para fins de subsistência, utilizaremos o espaço ecológico do território de energia que tem um fator de equivalência de 1,37 para transformação do resultado em global hectare (GHA).

O território de energia se define como áreas fictícias que devem ser reservadas para que a fauna e flora possam realizar suas atividades, sobreviver e propagar, ou seja, o espaço destinado a proteção da 
biodiversidade. É o espaço ecológico necessário para absorção do carbono $\left(\mathrm{CO}_{2}\right)$, evitando assim a sua concentração na atmosfera e o aquecimento global (PARENTE, 2007).

Cálculos utilizando essa ferramenta metodológica ainda são muito tímidos. No Brasil agora que se iniciaram as pesquisas. Na Amazônia são raros os trabalhos defendidos e, raríssimos publicados. SANTOS et al. (2013) contabilizou a alimentação urbana da cidade de Parintins por meio deste indicador, porém não encontramos trabalhos publicados sobre a contabilização de RSU por meio desse indicador.

O pensamento deste trabalho é contabilizar a pegada ecológica por meio dos passos utilizados no trabalho de vários autores (WACKERNAGEL et al., 1996; DIAS, 2002; PARENTE, 2007; SANTOS, 2013) que a utilizaram para diversas contabilizações ambientais.

A seguir demonstraremos as etapas utilizadas para o cálculo da pegada ecológica proposto neste estudo: (1) População, conforme dados fornecidos pelo IBGE (2010); (2) O volume de resíduos gerados em toneladas foi levantado junto a Secretaria de Limpeza Pública de Parintins (SEMOSP) e a empresa coletora TERCON; (3) O valor da emissão de $\mathrm{CO}_{2}$ em toneladas foi calculado obedecendo as seguintes normas; segundo Andrade (2006), 3 (três) libras de resíduos produz 1 (uma) libra de $\mathrm{CO}_{2}$ e 1 (uma) libra é igual a 0,45 kg, então $1,35 \mathrm{~kg}$ de resíduos produzem $0,45 \mathrm{~kg}$ de $\mathrm{CO}_{2}$. Sendo assim, entende-se que dividindo o total de resíduos gerados em toneladas por 1,35 e multiplicado o resultado por 0,45 encontra o total de $\mathrm{CO}_{2}$ emitido em toneladas; (4) O Ecological Footprint Method em hectare da população, referente à emissão de $\mathrm{CO}_{2}$, foi calculado partindo do princípio que 1 (uma) tonelada corresponde a $1.000 \mathrm{~kg}$ e que 1 (um) hectare de terra absorve 1 (uma) tonelada de $\mathrm{CO}_{2}$, portanto, o Ecological Footprint Method em hectare é igual ao valor da emissão de $\mathrm{CO}_{2}$ em tonelada; (5) O Ecological Footprint Method em hectare per capita, referente à emissão de $\mathrm{CO}_{2}$, foi calculado dividindo o Ecological Footprint Method em hectare da população $\mathrm{CO}_{2}$, item 03 pela população, item 01; (6) O Ecological Footprint Method em hectare da população, referente à emissão de $\mathrm{CO}_{2}+\mathrm{CH}_{4}$, foi calculado conforme dados registrados por Andrade (2006), onde consta que para um $\mathrm{kg}$ de $\mathrm{CO}_{2}$ também é gerado um $\mathrm{kg}$ de $\mathrm{CH}_{4}$. Então, para chegar ao resultado do item 06, foram multiplicados os resultados do item 04 por dois; (7) O Ecological Footprint Method em hectare per capita, referente à emissão de $\mathrm{CO}_{2}+\mathrm{CH}_{4}$, foi calculado através da multiplicação dos valores do item 05 por dois, seguindo o mesmo raciocínio do item 07; (8) O Ecological Footprint Method em global hectare, referente à emissão de $\mathrm{CO}_{2}+\mathrm{CH}_{4}$, foi calculado através da multiplicação do Ecological Footprint Method em hectare da população $\mathrm{CO}_{2}+\mathrm{CH}_{4}$, pelo Fator de Equivalencia 1,37 referente à bioprodutividade global da terra de energia; (9) O Ecological Footprint Method em global hectare per capita, item 09, referente à emissão de $\mathrm{CO}_{2}+\mathrm{CH}_{4}$, foi levantado através da divisão do resultado do item 08 pela população, item 01

A pesquisa obteve dados quantitativos junto a Secretaria Municipal de Limpeza Pública (SEMOSP) e Secretaria Municipal de Meio Ambiente (SEMMA) de Parintins que demonstrou uma produção diária RSU de 64 toneladas (2011), conforme também dados dos trabalhos de Filho et al. (2012) e Pessoa et al. (2013), chegando a 80 toneladas em 2013. Ao contrário, Picanço et al. (2014), encontrou para Parintins uma geração de resíduos sólidos de 1,56 kg/hab., um total de 110 toneladas diariamente. Porém, neste trabalho adotaremos os dados da SEMOSP. 


\section{RESULTADOS}

\section{Pegada Ecológica dos Resíduos Sólidos de Parintins}

Parintins é o segundo município mais populoso do estado do Amazonas. Tem os seus limites assim definidos: ao Norte com o Município de Nhamundá, ao Sul com o Município de Barreirinha, a Leste com o Estado do Pará (Município de Juruti) e a Oeste com o Município de Urucurituba. A área territorial de Parintins é de $5.952,378 \mathrm{Km}^{2}$ (IBGE, 2010).

Na hierarquia urbana da Amazônia, Parintins classifica-se como uma cidade média com dinâmica econômica externa, pois a atividade econômica responsável pelo seu dinamismo não agrega valor nem no local nem regionalmente (SHOR et al., 2011).

A área urbana tem 396 hectares disponíveis para o consumo e uso de seus habitantes. Quando dividimos a área total urbana pela população têm-se uma área per capita de 0,005 de hectares. Essa ainda não é a pegada ecológica, apenas a área disponível para cada habitante urbano (SANTOS et al., 2013).

A área de lixeira de Parintins ocupa uma superfície de 300 × 350 metros ou 10,5 hectares, a uma distância de 4,6 km do aeroporto da cidade trazendo risco aviário e problemas ambientais, pois localiza-se em meio a área urbana (TCE, 2012). Os problemas de resíduos sólidos precisam ser resolvidos no âmbito da gestão compartilhada com empreendedores e consumidores com responsabilidades conjuntas na redução do RSU (REVEILLEAU, 2007).

É um sério problema ambiental que necessita de um cálculo para quantificar em área (hectare) as emissões de $\mathrm{CO}_{2}$ e $\mathrm{CH} 4$ na atmosfera da quantidade produzida de resíduos sólidos nesta cidade. Nesse sentido, faz-se a seguir o cálculo da pegada ecológica da produção de resíduos sólidos de Parintins. O cálculo da quantidade de RSU (diário, mensal e anual) e a pegada ecológica da cidade de Parintins podem ser verificados nas Tabelas 1 e 2 .

Tabela 01: Toneladas de RSU de Parintins nos anos de 2011 e 2013.

\begin{tabular}{r|r|r|r}
\hline \multicolumn{1}{r|}{ Ano } & Dia (ton.) & Mês (ton & \multicolumn{1}{c}{ Ano (ton) } \\
\hline 2011 & 65 & 1.950 & 23.040 \\
\hline 2013 & 80 & 2.400 & 28.800 \\
\hline
\end{tabular}

Tabela 02: Pegada Ecológica dos RSU da cidade de Parintins.

\begin{tabular}{l|l|l|l|l|l|l|l} 
& \multicolumn{1}{|c|}{ População } & RSU (ton) & Co2 (ton) & Co2+CH4 (ton) & $\begin{array}{c}\text { (Co2+CH4) } \\
\text { per capita }\end{array}$ & $\begin{array}{c}\text { Gha } \\
\text { per capita } \\
\text { Total }\end{array}$ \\
\hline 2011 & 69.890 & 23.400 & 7.800 & 15.600 & 0,2233 & 0,3059 \\
\hline 2013 & 69.890 & 28.800 & 9.600 & 19.200 & 0,2747 & 21.380 \\
\hline
\end{tabular}

Por meio do valor diário total do resíduo solido coletados na cidade realizou-se a contabilização anual. Porém, esclarecemos que não consideramos as diferenças da quantidade produzida em determinadas épocas do ano. Por exemplo, no mês de junho em Parintins, esse valor tende a se elevar devido ao número de turistas, mesmo que por pouco sua permanência seja por pouco tempo nos três dias do festival folclórico dos Bois Bumbás. A alta temporada turística não foi considerada neste trabalho, a exemplo da cidade de Joiville que para o cálculo se considerou os meses de janeiro e fevereiro para alta e os meses de março a dezembro como baixa temporada (PARENTE, 2007). 
Portanto, para Santos (1991), "o espaço é resultado da ação dos homens sobre o próprio espaço, intermediados pelos objetos, naturais e artificiais". A pegada ecológica, assim, ao quantificar os desafios ecológicos pode contribuir com decisões políticas para o alcance de um mundo mais sustentável.

\section{DISCUSSÃO}

\section{Sociedade Urbana e os Resíduos Sólidos}

Na sociedade urbana são poucos que se sensibilizam com os problemas ambientais e em se tratando de RSU, nem mesmo o poder público se importa com a questão como requer hoje o conceito dos 3Rs (reduzir, reutilizar e reciclar). Compreendendo a cidade como uma área que demanda cada vez mais recursos naturais para sua manutenção, sobretudo, em relação ao consumo. Cada vez mais as sociedades urbanas consumem bens industrializados que em sua maior parte irão parar nas lixeiras públicas e reduzir os espaços destinados a biocapacidade do Planeta.

Atualmente, a média da Pegada Ecológica mundial é de 2,7 hectares globais por pessoa, enquanto a biocapacidade disponível para cada ser humano é de apenas 1,8 hectare global (gha/cap). Isso coloca a humanidade em grave déficit ecológico de 0,9 gha/cap, ou, expressado de outra forma, a humanidade consome um planeta e meio, excedendo em $50 \%$ a capacidade regenerativa do Planeta (WWF, 2010).

$\mathrm{Na}$ cidade de Parintins a pegada ecológica do RSU vem aumentando a cada ano. Atualmente, ainda não dispõe de um Plano Integrado de Gerenciamento de Resíduos Sólidos (PIGRS) e assim sem lugar definitivo para a construção do aterro sanitário. Um impasse que perdura há anos sem que se concretize soluções para questão. Dessa maneira, o lixão na cidade está sobrecarregado, causando diversos danos à saúde da população, sobretudo no período do verão que constantemente pega fogo dispersando fumaça toxica provenientes de materiais como pilhas, isopor, plásticos entre outros gases que não estão aqui, neste cálculo, considerados.

A exemplo de Parintins, as principais cidades do estado do Amazonas ainda se encontram com lixeiras a céu aberto ou nos chamados lixões (TCE, 2012). Estes espaços antiecológicos emitem CO2 e CH4 para atmosfera sem que haja um esforço de contabilizar o problema. Neste trabalho, pretende-se fazer essa contabilidade ambiental para a cidade de Parintins.

O Global Hectare (gha) é a pegada ecológica. Seus resultados per capita e total demonstram a pressão sobre a natureza. Presta-se para comparações com outros lugares do mundo e padroniza a pegada ecológica em uma única unidade de medida (gha). Dessa maneira, elaboramos um quadro da pegada ecológica anual das cidades de Parintins e Florianópolis, conforme Tabela 03, abaixo:

Tabela 03: Comparação da pegada ecológica de Parintins e Joinvile.

\begin{tabular}{c|c|c|cc|c}
\hline CIDADE & ANO & POPULAÇÃO & CO2 + CH4 & GHA TOTAL & GHA per capita \\
\hline $\begin{array}{c}\text { JOINVILE } \\
\text { (Alta temporada) }\end{array}$ & 2005 & 968.355 & $8.118,77$ & $11.122,57$ & 0,0114 \\
\hline $\begin{array}{c}\text { JOINVILE } \\
\text { (Baixa temporada) }\end{array}$ & 2005 & 386.913 & $6.437,13$ & $8.818,87$ & 0,0227 \\
\hline PARINTINS & 2013 & 69.890 & $19.200,00$ & $26.304,00$ & 0,3763 \\
\hline
\end{tabular}


No caso parintinense a pegada ecológica $(0,3763)$ per capita é maior do que a cidade de Joinvile-SC, mesmo considerando a baixa e alta temporada de fluxo turístico (0, 0227 e 0,0114 gha), assim como, é alta a sua pegada total (26.304,00 gha) em comparação a Joinvile (11.122,57 gha) na alta temporada turística.

Quanto maior a população de um determinado ambiente urbano, maior a pressão sobre os recursos naturais. No caso de Parintins (AM) ainda que com uma população menor que Joinville (SC) apresenta uma alta pegada ecológica devido a vários fatores como: falta de um plano de gerenciamento dos RSU; consumo grande de produtos industrializados e, com isso, aumento da geração de RSU e menor área urbana disponível para cada habitante.

A contabilidade ambiental tende a ser negativa se a área (hectare) disponível por cada cidadão, por exemplo, se torna menor do que a área disponível para absorção da poluição gerada pelo sistema urbano. A área per capita (0,005 hectares) de Parintins é menor que a pegada ecológica de 0,3763 dos RSU, demonstrando a insustentabilidade da produção de resíduos sólidos na cidade e necessitando urgentemente políticas públicas que considerem essa pressão ecológica aqui mensurada.

Portanto, a mensuração dos RSU por meio desse indicador de sustentabilidade consegue demostrar a pressão ecológica sobre os ecossistemas, pois contabiliza em $\mathrm{CO} 2+\mathrm{CH} 4$ a quantidade produzida, e, transforma em global hectare (gha), o espaço necessário para absorver essa poluição.

\section{CONSIDERAÇÕES FINAIS}

A pegada ecológica é um parâmetro de avaliação ambiental e se presta para cumprir metas estratégicas para o futuro, ou seja, mostrar de maneira sustentável. A pegada ecológica dos resíduos sólidos aqui calculados no período estudado (2011 e 2013) representa um impacto negativo sofrido pelo ecossistema. Uma dívida ecológica que está sendo paga pela sociedade como um todo, sobretudo nos períodos em que a fumaça toxica da lixeira invade a cidade. Outros impactos estão relacionados a contaminação dos solos, lençol freático, doenças diversas e sobretudo, a emissão de $\mathrm{CO}_{2}$ (dióxido de carbono) e $\mathrm{CH}_{4}$ (metano).

O PIGRS precisa ser efetivado na cidade de maneira urgente como parte da adequação municipal a lei dos resíduos sólidos urbanos, prorrogada para 2016. A sua implementação conseguiria instalar o aterro sanitário da cidade e colocar em pratica o conceito da logística reversa que evitaria, no momento, a queima de RSU altamente poluidores do ar e que vem causando problemas respiratórios sérios na população. Assim, instituir o conceito de logística reversa auxiliaria em acordos setoriais e inclusão socioeconômica dos catadores e a destinação adequada dos RSU.

A população precisa urgente participar deste plano com colaboração nos planos de educação ambiental para a sensibilidade com o trato desta questão ambiental, no âmbito, sobretudo dos espaços formais e não formais. O consumo precisa ser sustentável. Caso contrário, estaremos caminhando para o "esgotamento do capital natural mais rápido, do que sua capacidade de renovação. Esse déficit é mantido por meio da degradação ambiental e da miséria de muitos povos" (DIAS, 2002; ANDRADE, 2006). 
O cálculo da pegada ecológica demonstra o aumento da quantidade de $\mathrm{CO} 2+\mathrm{CH} 4$ de 15.600 para 19.200 toneladas não é desprezível para esse centro urbano e requer se pensar novas maneiras de analisar o aquecimento global na região amazônica, não somente pela ótica das queimadas, mas também pela geração de RSU de suas cidades e outros tipos de impactos ecológicos.

\section{REFERÊNCIAS}

ANDRADE, B. B.. Turismo e sustentabilidade no município de Florianópolis: uma aplicação do método da pegada ecológica. Dissertação (Mestrado em Administração) Universidade Federal de Santa Catarina, Florianópolis, 2006.

CAVALCANTI, C.. Desenvolvimento e Natureza: estudo para uma sociedade sustentável-São Paulo: Cortez, 1998.

DIAS, G. F.. Pegada ecológica e sustentabilidade humana. São Paulo: Gaia, 2002.

FILHO, C.; TEIXEIRA, G.. A gestão de resíduos sólidos em Parintins/AM à luz da Política Nacional de Resíduos Sólidos. Dissertação (Mestrado em Ciências Ambientais) Universidade Federal do Amazonas, Manaus, 2012.

FIRMINO, A. M.; SANTOS, H. N.; PINA, J. H. A.; RODRIGUES, P. O.; FEHR, M.. A relação da pegada ecológica com o desenvolvimento sustentável: cálculo da pegada ecológica de Toribaté. Caminhos de Geografia, Uberlândia, v.10, n.32, p.41-56, 2009.

IBGE. Instituto Brasileiro de Geografia e Estatística. Censo 2010. Brasília: IBGE, 2010.

PARENTE, A.. Indicadores de Sustentabilidade Ambiental: Um Estudo do Ecological Footprint Method do Município de Joinville - SC. 2007.

PESSOA, A.; ORESTE, L.; FILHO, A. M.. O Problema do Lixo em Parintins (AM) e a Inserção da Associação dos Catadores na Coleta Seletiva. 2013.

PICANÇO, S. F.; TELLO, J. C. R.; COELHO, L. M.. Caracterização física dos resíduos sólidos urbanos produzidos na cidade de Parintins-AM. Revista Ibero-Americana de Ciências Ambientais, v.5, n.2, 2014.

QUEIROZ, T. D.; REIS, B. C.; BRAGA, M. M. V.; RODRIGUEZ, I. C. A. G.. Temais transversais \& Conteúdos normais: Proposta prática de construção de conhecimento Transversal: 1o ciclo. São Paulo: Didática Paulista, 2000.

REVEILLEAU, A. C. A.. A Política e gestão compartilhadas de resíduos sólidos no âmbito do poder público, do empreendedor e do consumidor: responsabilidade socioambiental e sua implementação. Dissertação (Mestrado em Gestão Ambiental) - Pontifícia Universidade Católica de São Paulo, São Paulo, 2007.

RIBEIRO, E. M.. Prospectiva e sustentabilidade do ecoturismo: uso da técnica de construção de cenários no estado do amazonas. Tese (Doutorado em Desenvolvimento Sustentável) - Universidade de Brasília, Brasília, 2013.
SANTOS, M.. Metamorfoses do Espaço Habitado. 2 ed. São Paulo: Hucitec, 1991.

SANTOS, M.; SILVIA, A.. Alimentação urbana e a pegada ecológica do consumo de carne bovina na cidade de Parintins. Acta Geográfica, v.7, n.14, p.45-53, 2013.

SICHÉ, R.; AGOSTINHO, F.; ORTEGA, E.; ROMEIRO, A.. Índices versus indicadores: precisões conceituais na discussão da sustentabilidade de países. Ambiente e Sociedade, v.10, n.2, p.137-148, 2007

SCHOR, T.; OLIVEIRA, J. A.. Reflexões metodológicas sobre o estudo da rede urbana no amazonas e perspectiva para a análise das cidades na Amazônia brasileira. Acta Geográfica, Boa Vista, p.15-30, 2011.

TCE. Tribunal de Contas do Estado do Amazonas. Panorama dos resíduos sólidos em nove Municípios do Amazonas: Relatório de Vistorias Operacionais do TCE/AM. 2012.

WACKERNAGEL, M.; REES, W. E.. Our Ecological Footprint: reducing human impact on the earth. Quebec: New Society Publishers, 1996.

WWF. World Wide Fund. Living Planet Report 2010 Zurique: WWF/ZSL/GFN, 2010. 